\title{
An Imaginary Friend that Connects with the User's Emotions
}

\author{
Sofia Reis, Nuno Correia \\ CITI, Faculdade de Ciências e Tecnologia da \\ Universidade Nova de Lisboa \\ 2829-516 Caparica - Portugal \\ +351212948300 \\ se.reis@fct.unl.pt,nmc@di.fct.unl.pt
}

\begin{abstract}
In this paper we present a metaphor of an Imaginary Friend that walks along with the user. Due to a special bond with the user, the Imaginary Friend can see the emotions that the human companion is feeling glowing around her. As the user moves around she leaves behind emotion cookies that the Imaginary Friend will collect and treasure. In order to enable rich and playful interactions in the virtual-real continuum the implementation of the Imaginary Friend responds to motion, to the electrodermal activity and resorts to GPS coordinates. The Imaginary Friend was tested with several users to evaluate the connection between humans and this creature that lives in the border that separates imagination and reality.
\end{abstract}

\section{Categories and Subject Descriptors}

J.m Computer Applications:Miscellaneous. H5.m. Information interfaces and presentation (e.g., HCI): Miscellaneous.

\section{General Terms}

Human Factors.

\section{Keywords}

Imaginary Friend, Companion, Electrodermal Activity, Pico projector, Accelerometer, Affective Wearables.

\section{INTRODUCTION}

Sometimes, one wants a friendly shoulder to lean on or someone with whom to share the good and the bad times. Here, we propose a metaphor of an imaginary friend ${ }^{1}$ who accompanies the user and to whom the user can confide her emotions.

Imaginary friends, though nonexistent, provide many advantages to their human companions. According to Eva Hoff, imaginary friends can help one get through difficult or stressful times [1] and children with imaginary friends are significantly more creative [2]. Imaginary friends are common among children. Approximately $50 \%$ of children aged between 5 and 12 years old have experiences with imaginary friends [3]. Perhaps many adults also have imaginary friends but lack the courage to assume it because they feel it is too childish a thing for their age. That is not the case of adult fiction writers, as $92 \%$ of them experience the

Permission to make digital or hard copies of all or part of this work for personal or classroom use is granted without fee provided that copies are not made or distributed for profit or commercial advantage and that copies bear this notice and the full citation on the first page. To copy otherwise, or republish, to post on servers or to redistribute to lists, requires prior specific permission and/or a fee.

Full presentation, ACE'2011 - Lisbon, Portugal

Copyright 2011 ACM 978-1-4503-0827-4/11/11 ...\$10.00.

${ }^{1}$ For a video about the project please go to http://www.youtube.com/watch?v=8XvsUfU6jb4 illusion that their characters have independent thoughts, words or actions, as if they were real persons [4].

Countless people write to an imaginary friend that exists only in the pages of a diary. One of the most famous of these imaginary friends is Kitty, who accompanied Anne Frank through the difficult times in the attic during the Second World War. Another well know imaginary friend is Hobbes from the Calvin and Hobbes cartoon books by Bill Watterson. Hobbes is a tiger that accompanies Calvin and discusses everyday matters with him. Hobbes is really just a stuffed tiger but, for Calvin, Hobbes is much more than a mere toy. He is alive and has feelings, just like a real person.

The Imaginary Friend proposed here walks by the side of the user. The user can confide the emotions she is feeling to the Imaginary Friend. This concept is further detailed in Section 2 and the user tests can be found in Section 3. In Section 4 a proposal to expand the Imaginary Friend to a multiplayer game is presented.

\section{THE IMAGINARY FRIEND}

Our objective is to create an Imaginary Friend that accompanies the user and keeps her company (Figure 1 (a)).

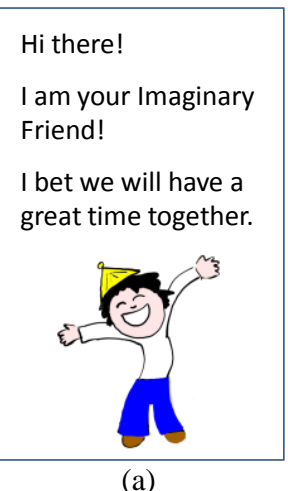

As you walk around I shall collect the emotion cookies you leave behind.

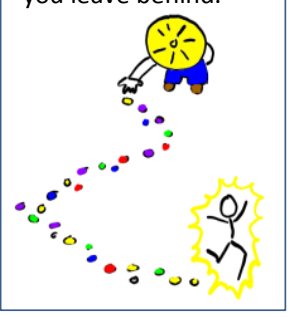

(c)

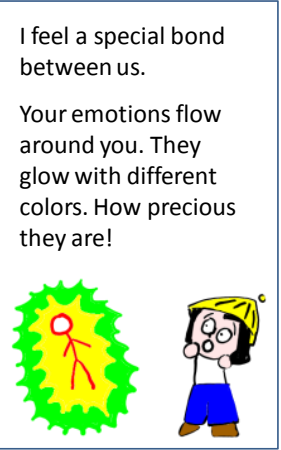

(b)

I will safe keep all your emotion cookies, but I will need your help to catalog them.

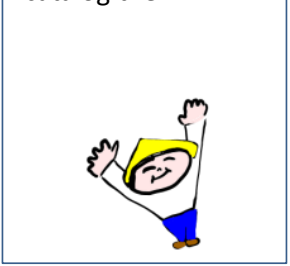

(d)
Figure 1. The Imaginary Friend's concept. 
This Imaginary Friend has a special bond with the user and can feel what the user is feeling (Figure 1 (b)). As the user walks around, in her everyday life, she leaves behind emotion cookies (Figure 1 (c)). These cookies are invisible to creatures from the real world. However, the Imaginary Friend, who lives in the border between the real world and the imagination world, can see those emotion cookies and will store them all in a jar. To know the emotion that corresponds to each cookie the Imaginary Friend will need the user's help. Each time a cookie is collected, the Imaginary Friend will question the user to discover to what sort of emotion that cookie corresponds (Figure 1 (d)).

Subsection 2.1 and subsection 2.2 explain how the Imaginary Friend was implemented. Subsection 2.1 focuses on the walking activity. Subsection 2.2 focuses on the emotion cookies.

\subsection{Walking along with the user}

Our Imaginary Friend accompanies the user. When the user looks down the friend is there. The Imaginary Friend is projected on the floor using a pico projector attached to the user's backpack shoulder strap (Figure 2). The projector is connected to a mobile phone that runs the interactive application. The user also wears a bracelet with an electrodermal activity sensor. This sensor is used to measure the arousal of the user, as is further explained in Subsection 2.2. In Figure 3 a close up of the Imaginary Friend is presented.

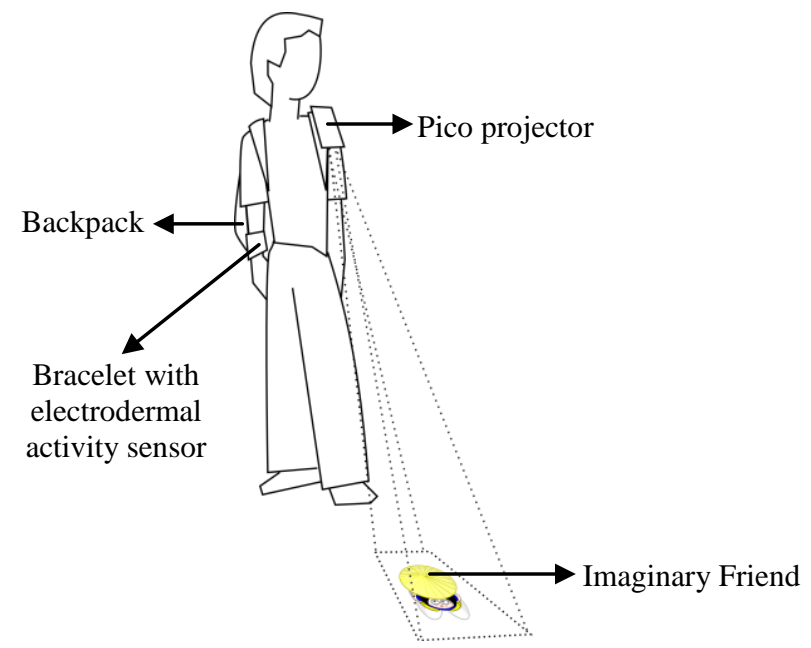

Figure 2. Illustration of the user with the Imaginary Friend.

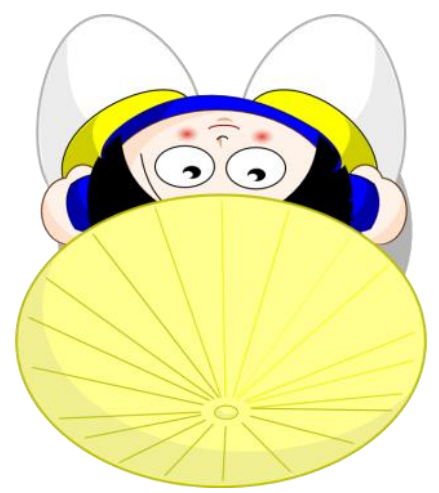

Figure 3. Close up of the Imaginary Friend.
Figure 4 shows two photographs of two users with the Imaginary Friend. These users have a pico projector attached to their backpack shoulder strap, as represented in Figure 2. The users are also wearing the bracelet, with the electrodermal activity sensor, on their right wrist.
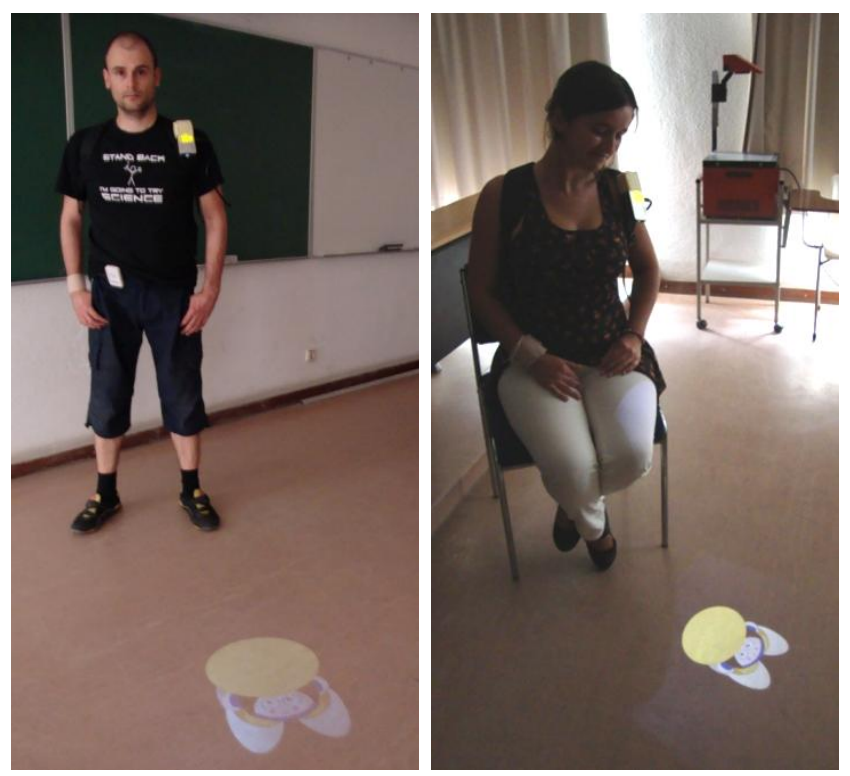

Figure 4. Two users with the Imaginary Friend. The Imaginary Friend can be seen, projected on the floor, by the side of each user.

When the user is walking, the Imaginary Friend is also walking besides the user. In the animation of the imaginary friend, the hands and feet move back and forth to convey the impression of a human being walking (Figure 5).

The Imaginary Friend is seen from a top view as if he is someone shorter than the user. The background is black, so that in that area no colors are projected. This way, we tried to create the illusion that the Imaginary Friend is really standing on the floor. We considered including a grass background but the grass would not feel like part of a real floor. A black background was therefore considered the best choice. The Imaginary Friend has very light colors because darker colors were harder to see in the projection.
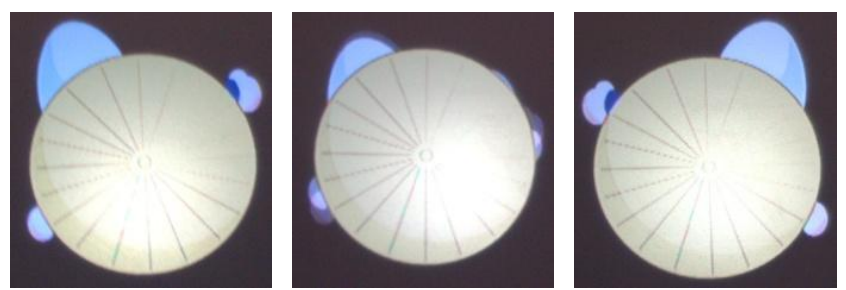

Figure 5. Projected image of the Imaginary Friend while the user is walking. Hands and feet move back and forth.

When the user is walking, the Imaginary Friend assumes she is too busy and just walks besides the human companion keeping company.

When the user stops walking, the Imaginary Friend also stops and looks up to convey that he is paying attention to what the user is doing (Figure 6). We detect if the user is standing or walking by analyzing the accelerometer data on the mobile phone. 


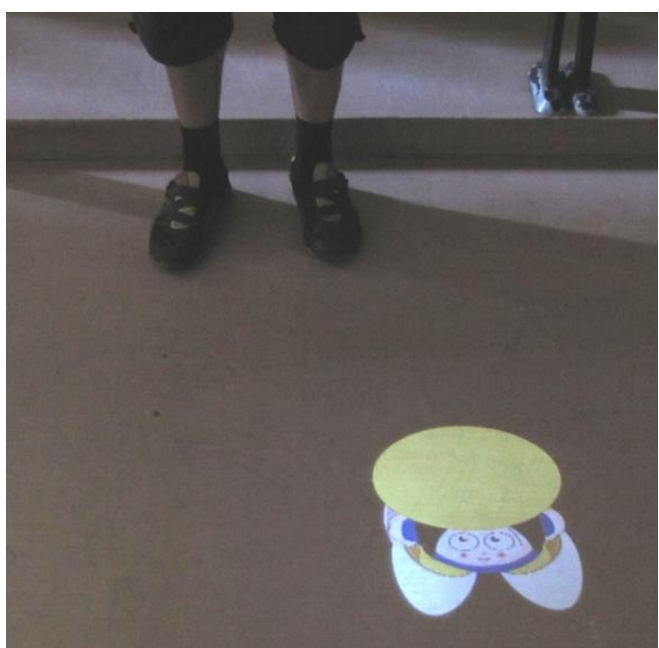

Figure 6. Projected image of the Imaginary Friend while the user is standing still.

\subsection{Connecting with the user's emotions}

The Imaginary Friend automatically detects changes in the arousal of the user. Arousal corresponds to a change in conductance at the surface of the skin due to an external or internal stimuli experienced by the user [5]. The user wears a bracelet with the electrodermal activity sensor on her wrist (Figure 2). The electrodermal activity sensor is attached to the user's wrist. The bracelet is used to prevent the sensor from falling or from displacing due to the user's movement. In Figure 7, the bracelet is not strapped and the sensor can be seen on the user's wrist. In Figure 8 the bracelet is strapped. The sensor is connected, with wires, to a PLUX device [6] that transmits the data, via Bluetooth, to the mobile phone. The PLUX device can be attached to a belt, placed inside a pocket or inside the backpack. The mobile phone is connected to the pico projector, in order to project the Imaginary Friend on the floor. We attached the pico projector to the backpack shoulder strap with tape. Some of that yellow tape can still be seen in Figure 7.

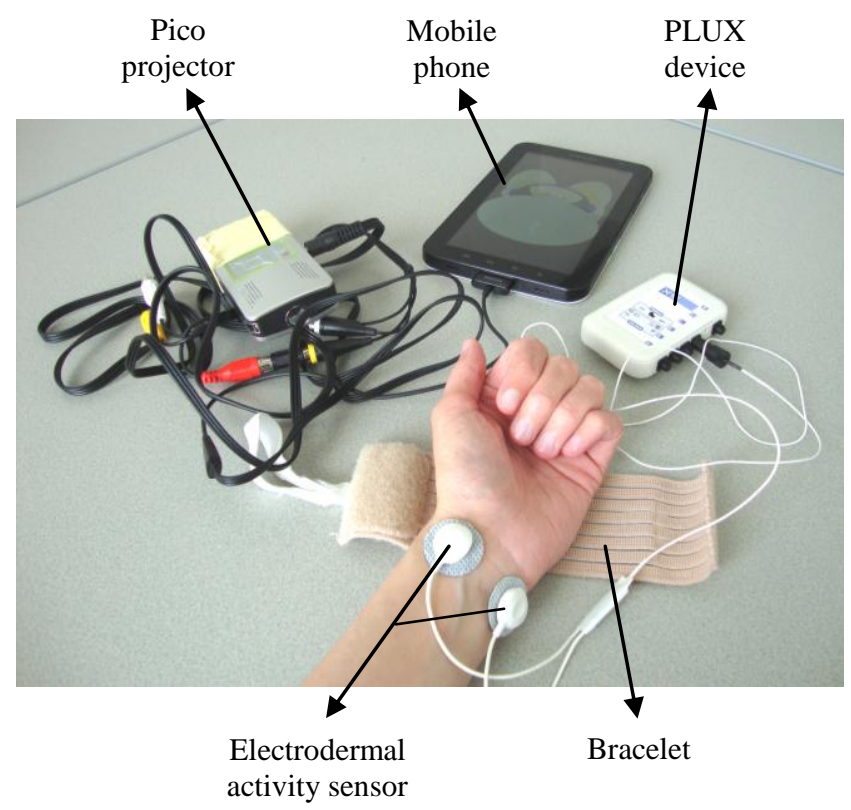

Figure 7. All the devices used in the Imaginary Friend.

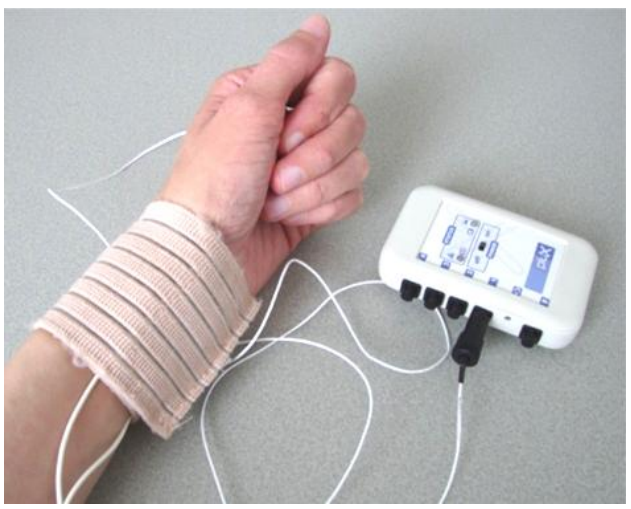

Figure 8. The bracelet, with the sensors underneath, on the left, connected to the PLUX device, on the right.

When there is a significant increase or decrease in the arousal, the external or internal stimuli behind that change might have caused an alteration in the emotion the user is feeling. To discover what emotion the user is feeling, the Imaginary Friend will pose a question and present several emotion tags (Figure 9 (a)). The phone beeps and vibrates to warn the user that the Imaginary Friend is posing a question. The available emotion tags are Happy, Sad, Afraid, Angry, Annoyed, Bored, Calm, Confused, Depressed, Distracted, Empty, Energetic, Envious, Hopeful, Lonely, Loved, Sick and Tired. To attribute a color to each emotion tag we used an adaptation of the colors proposed in [7], [8] and [9]. Happy and Sad are emotions easy to interpret, so we placed them near the top left corner of the screen. The rest of the emotions are organized in alphabetical order. To choose an emotion tag the user presses the corresponding button on the mobile phone screen (Figure 9 (b)).

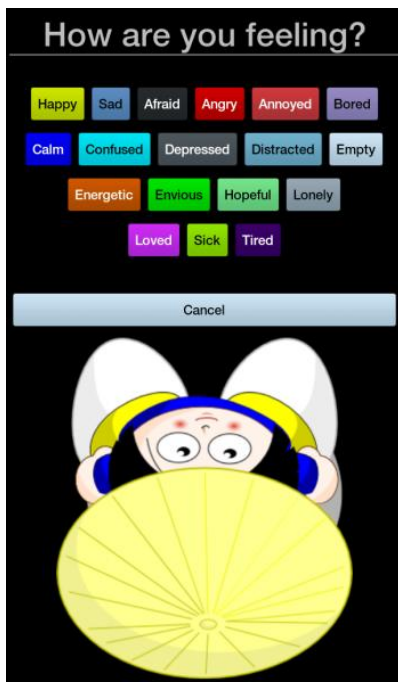

(a) Emotion tags

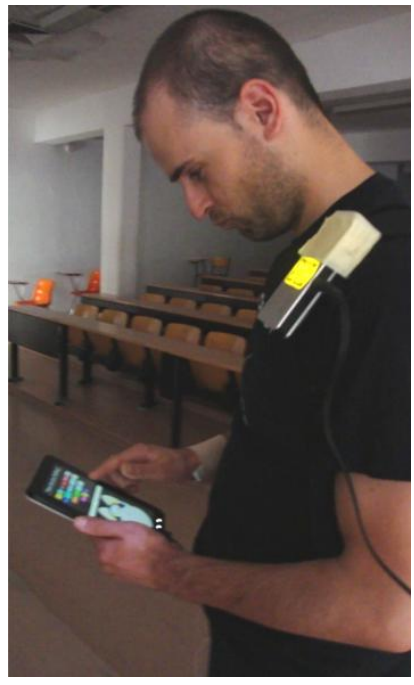

(b) User selecting an emotion tag
Figure 9. The Imaginary Friend asks how the user is feeling.

After the user selects an emotion tag, an emotion cookie is left on the ground. The cookie is the same color as the color of the selected emotion tag. In the case represented in Figure 10 (a) the cookie is yellow because the user selected the emotion tag Happy. The emotion cookie already inside the jar has the same color as the previously selected emotion tag. In this specific case it was the Energetic emotion tag. The Imaginary Friend picks up the emotion cookie and stores it inside a jar (Figure 10 (b)). The most 
recent emotions are near the opening of the jar, on the left side of the picture. The most ancient emotions are at the bottom of the jar on the right side of the picture. The animation associated with the process of storing the emotion cookie is projected on the floor.

By looking at the most frequent colors inside the jar the user can have an idea of what emotions have dominated her recent life.

The jar in Figure 10 (b) is an edited photo of a real jar. This is meant to cause a contrast with the cartoon style of the Imaginary Friend as the jar symbolizes a connection with reality. The user's emotions are real and so they are stored inside a real jar by the Imaginary Friend.

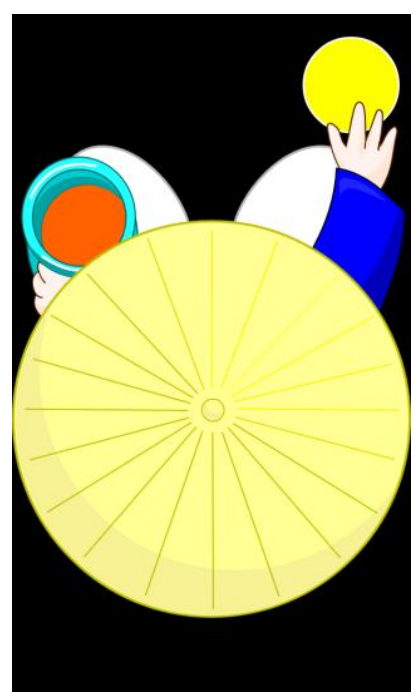

(a) Retrieving the emotion cookie left behind by the user

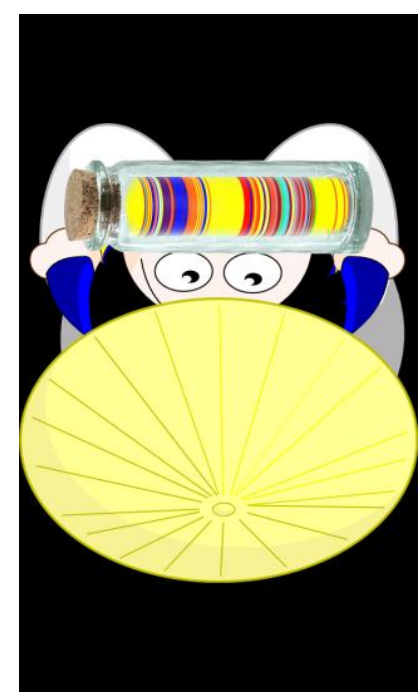

(b) Jar with the stored emotion cookies

\section{Figure 10. Collecting and storing the emotion cookies.}

Besides the jar, there are other ways of consulting past emotions. The user can consult a list with all the past emotions ordered by descending chronological order (Figure 11). Each line shows the color and the name of the emotion tag selected by the user, when answering to the Imaginary Friend. Each line also indicates when and where the emotion cookie was collected.

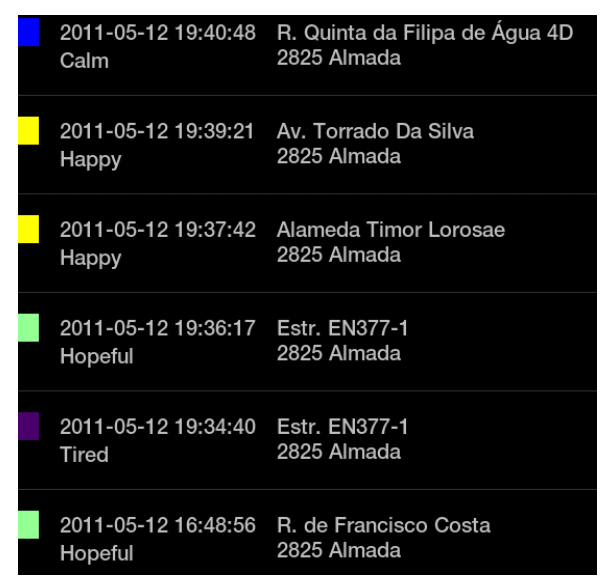

Figure 11. List of past emotions experienced by the user.

The user can also visualize, in a map, the places where each emotion cookie was collected by the Imaginary Friend (Figure 12). Each circle, in this map, represents an emotion cookie. The current position of the user is represented by a red figure.
When the user clicks a line, in Figure 11, the list will disappear and the map will appear, centered at the point where that emotion cookie was collected.

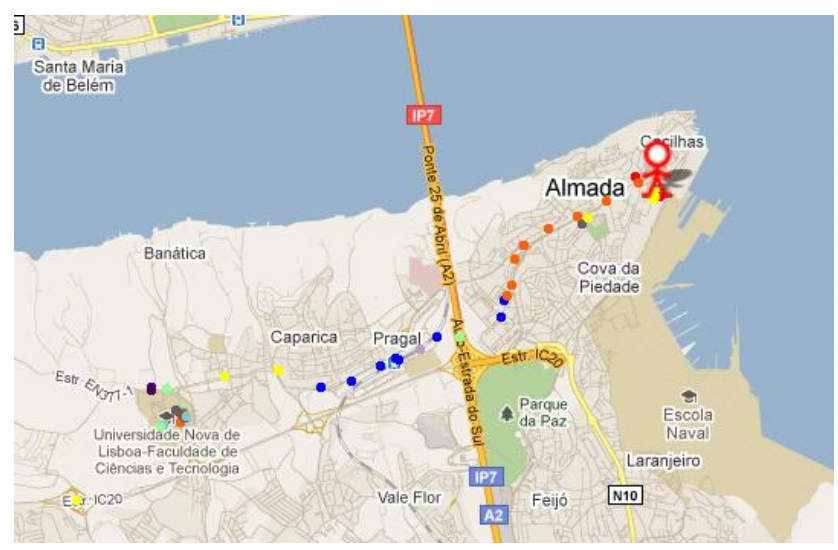

Figure 12. Places where the emotion cookies were collected by the Imaginary Friend. The current position of the user is represented by a red figure.

\section{TESTING THE IMAGINARY FRIEND}

The Imaginary Friend was tested with 11 users. The youngest user is 15 years old and the oldest user is 58 years old. The average user age is 36,5 years old. 5 of the users are females and the remaining 6 users are males. The users were questioned, with a paper survey, after they used the Imaginary Friend application. The survey was previously tested.

When the Imaginary Friend asks the user what emotion she is feeling (Figure 9) the mobile phone vibrates and beeps. We wondered if this was enough to captivate the user's attention. 54\% of the users agreed or strongly agreed that they could clearly hear the beep. $27 \%$ of the users were completely unable to hear the beep or were hardly able to hear it (Figure 13). Some users complained that it was difficult to hear the beep when they were talking with other people or immersed in a noisy environment. However, almost all users were able to feel the phone vibrating when the Imaginary Friend posed a question (Figure 14). During the time that the users tested the application, the Imaginary Friend asked them what emotion they were feeling a total of 270 times. 237 answers were collected. Users were, therefore, able to answer $87,8 \%$ of the Imaginary Friend's questions. This seems to indicate that the beep combined with the vibration of the mobile phone was an efficient way to captivate these users' attention. Still, one user suggested the use of a flashing light, similar to a siren of a police car, to more effectively warn the user that the Imaginary Friend is posing a question. Another user suggested that instead of beeping the mobile phone could play a recording asking the user how she felt. The user argued that would be quite more personal than a simple electronic beep.

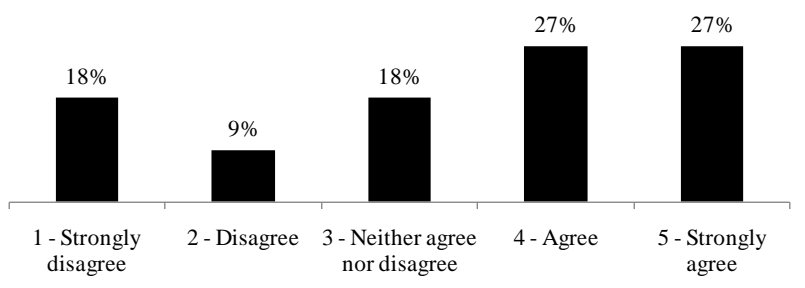

Figure 13. I heard the mobile phone beeping clearly, when the Imaginary Friend asked me what I was feeling. 


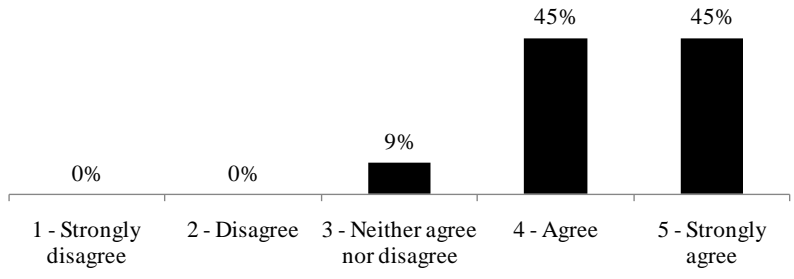

Figure 14. I felt the mobile phone vibrating, when the Imaginary Friend asked me what I was feeling.

$82 \%$ of the users agreed or strongly agreed that they were feeling a strong emotion or that the emotion they were feeling was changing when the Imaginary Friend asked them how they were feeling (Figure 15). 55\% of the users also thought the Imaginary Friend questioned them the appropriate number of times. The remaining $45 \%$ think the Imaginary Friend asked them how they were feeling many times (Figure 16). We consider this an important result because if the Imaginary Friend never or hardly poses questions the users may perceive the application as being unresponsive. If the questions pop up too often, the application may be perceived as annoying or as an unwelcome disruption in the users' daily activities.

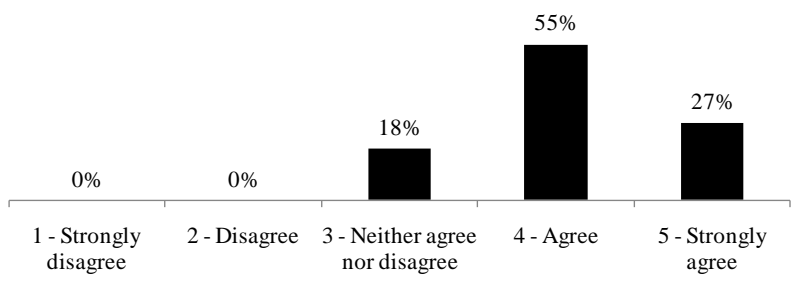

Figure 15. I was feeling a strong emotion or the emotion I was feeling was changing when the Imaginary Friend asked how I was feeling.

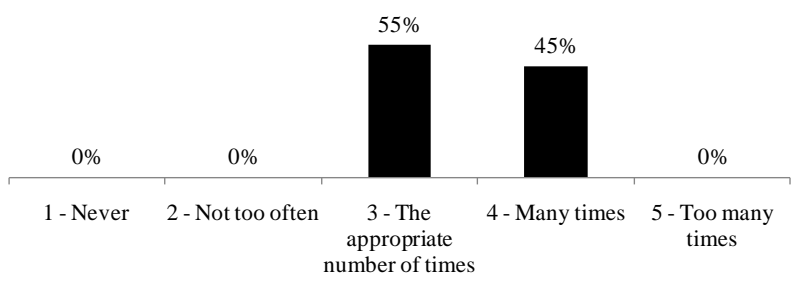

Figure 16. How many times did the Imaginary Friend ask you how you were feeling?

To inform the Imaginary Friend how she is feeling the user presses a button with an emotion tag on the mobile phone. $64 \%$ of the users considered that some extra emotion tags were needed (Figure 17). 36\% of the users complained that a button with the emotion tag Nervous or Anxious was missing. 18\% wanted a button with the emotion tag Focused and another $18 \%$ wanted a button with the emotion tag Hungry. One user asked for a better way of organizing the emotions and suggested ordering them by levels of intensity. This is an interesting suggestion but we found it hard to implement because the same emotion may have different levels of intensity. For example, a person may be a bit distracted or completely distracted. So it is difficult to know where distracted should be placed in that gradient. Adding emotion tags to the ones presented in Figure 9 (a) also presents some additional problems. The greater the number of emotion tags available, the harder it will be for the user to find the one she wants. Besides, the greater the number of emotion tags, the harder it will be to choose a unique color for them all.

Another aspect we were concerned with was the emotional connection users develop with the Imaginary Friend. If the users feel an emotional connection with the Imaginary Friend then perhaps those users will be more inclined to answer how they are feeling. 55\% of the users felt an emotional connection with the Imaginary Friend and $27 \%$ of them felt an intense emotional connection with the Imaginary Friend. None of them strongly disagreed or disagreed feeling an emotional connection with the Imaginary Friend (Figure 18). This suggests that none of the users felt antipathy for the Imaginary Friend.

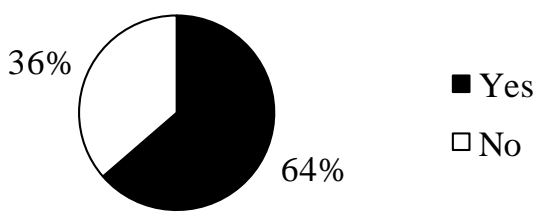

Figure 17. Did you feel the need for any more emotion tags besides the ones that were presented by the Imaginary Friend?

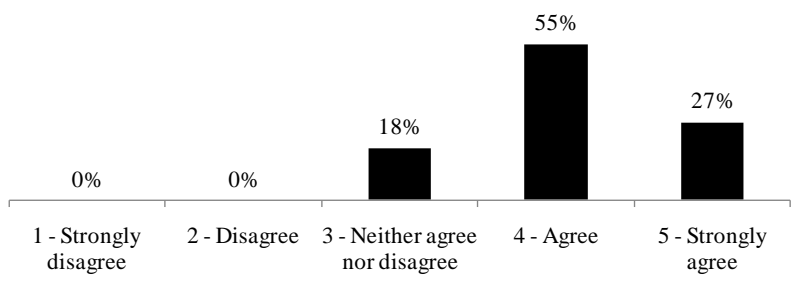

Figure 18. I felt an intense emotional connection with the Imaginary Friend.

The users could visualize their past emotions in three different ways: inside a jar (Figure 10 (b)), in a list (Figure 11) or in a map (Figure 12). 90\% of the users strongly agreed that they liked visualizing the past emotions as emotion cookies inside the jar, $71 \%$ strongly liked the map and $44 \%$ strongly liked the list (Figure 19). The jar was therefore the most favored form of visualization by the users. This form of visualization was the only one that was directly linked to the Imaginary Friend as the character held up the jar for the user to see. Both the list and the map occupied the whole screen of the mobile phone and the Imaginary Friend was not visible. This might have influenced the results.

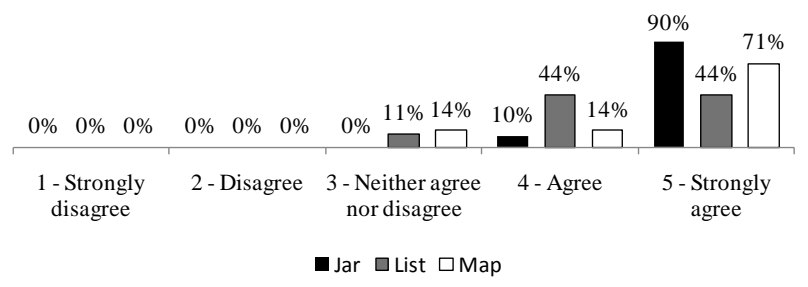

Figure 19. I liked this form of visualizing my past emotions.

Most of the users found it both useful and fun to register their emotions (Figure 20). In what refers to using an application that can automatically detect strong emotions or changes in the emotional state, the inquired users found it more fun than useful 
(Figure 21). 91\% of the users think it is fun to use such an application but only $45 \%$ believe it is useful.

$90 \%$ of the users agree or strongly agree that the Imaginary Friend can help them think about their emotions and take important conclusions about their lives (Figure 22). So, when the users were asked, simply, if it was useful to register their emotions, $63 \%$ of them agreed or strongly agreed (Figure 20) and when we asked them if it was useful to use an application that automatically detects strong emotions or when the emotions are changing the percentage of users who agreed or strongly agreed lowered to $45 \%$ (Figure 21). However, when a specific advantage was mentioned, thinking about their past emotions, some users seemed to reconsider and the percentage that agreed or strongly agreed increased to $90 \%$.

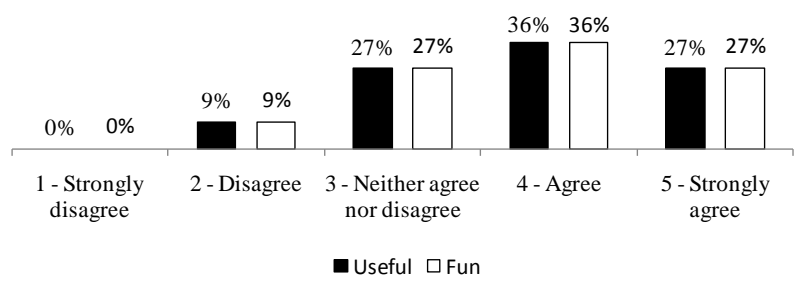

Figure 20. It is useful / fun to register my emotions.

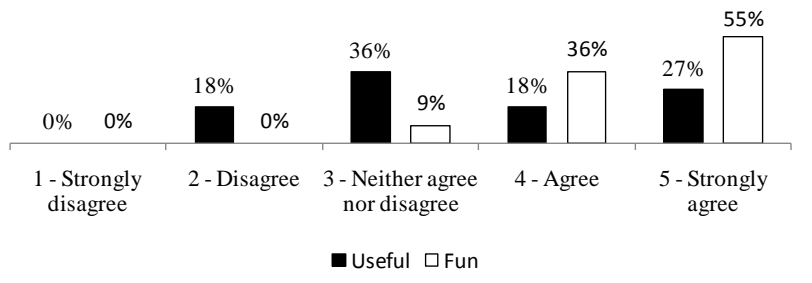

Figure 21. It is useful / fun to use an application that automatically detects when I am feeling a strong emotion or when my emotions are changing.

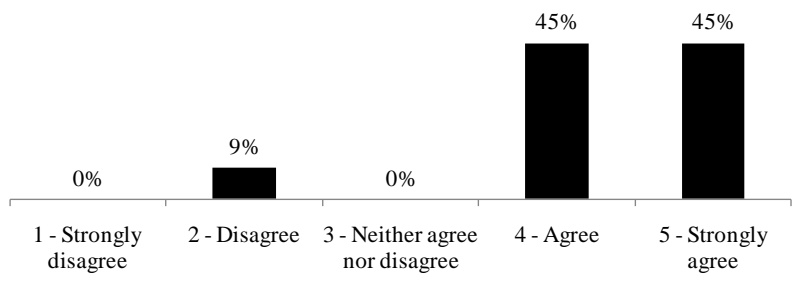

Figure 22. The Imaginary Friend can help me think about my emotions and take important conclusions about my life.

If they could share their answers to the Imaginary Friend, $18 \%$ of the users would share those answers with everybody, $64 \%$ would share their answers with friends only and $18 \%$ would not share their answers (Figure 23).

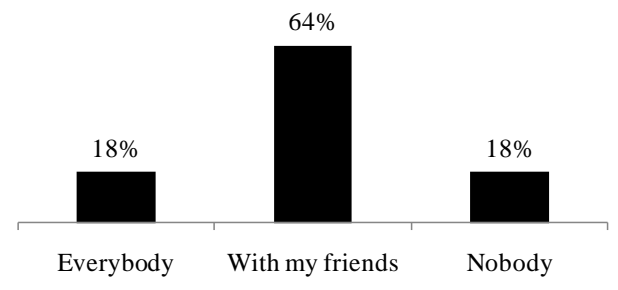

Figure 23. With whom would you share your answers to the Imaginary Friend?
As for the area where to place the electrodermal activity sensors, $67 \%$ of the users favored the wrist while the remaining $33 \%$ preferred the fingers via some device that looked like a ring. One of the users added that she would like the device to be as discrete as possible.

All of the users agreed or strongly agreed it was very easy interacting with the Imaginary Friend (Figure 24). All of them would recommend the Imaginary Friend to their friends.

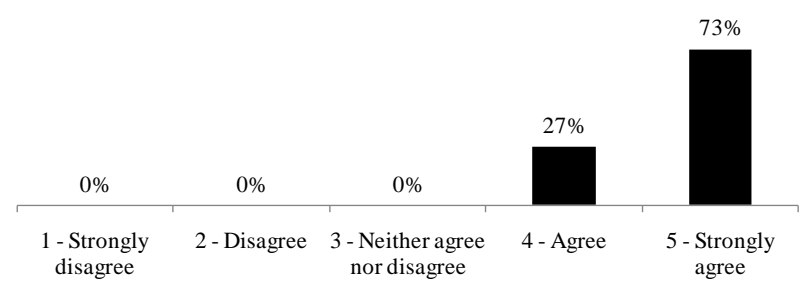

Figure 24. It was very easy interacting with the Imaginary Friend.

When the users were asked specifically if it was easy to answer to the Imaginary Friend in their everyday life, $81 \%$ of them agreed or strongly agreed it was very easy to do so (Figure 25). Even though the Imaginary Friend is projected on the floor, via the pico projector, to choose an emotion tag it is necessary to press a button in the mobile phone and that operation may be disruptive as it draws the attention away from the projection. Perhaps, using voice would be a better way to express the emotions and this will be tested in future work.

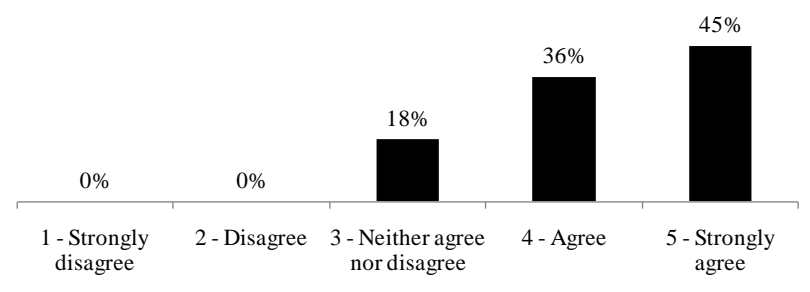

Figure 25. It was easy to conciliate the answers to the Imaginary Friend with my everyday life.

The users were asked to choose an expression that better describes their experience with the Imaginary Friend. $73 \%$ thought it was fun. $55 \%$ considered it simple. For $45 \%$ of them it was an easy to use, pleasant or innovative experience. Finally, 27\% considered it useful or motivating. The expressions are an adaptation of the Product Reaction Cards [10]. The users could choose as many expressions as they wanted.

\section{IN A NOT SO DISTANT FUTURE...}

The Imaginary Friend resorts to both a pico projector and to an electrodermal activity sensor. Pico projectors are already available in many places. As far as we know, there are no mobile phones with electrodermal activity sensors. However, there are already a few commercial versions available. We used a PLUX device [6]. The Q Sensor is another example of a commercially available device that measures emotional arousal [11]. In a not so distant future, devices that measure electrodermal activity may become more common and the Imaginary Friend proposed here may thus become a viable commercial proposal for the general public. If several people use devices that measure the electrodermal activity it will be possible to resort to competition to better motivate users to tell the Imaginary Friend how they are feeling. Our proposal is to populate the Map in Figure 12 with emotion castles. When a large number of emotion cookies are close together a castle will 
rise. The castle flag is the same color of the most frequent emotion in that area (Figure 26). A castle belongs to all players who contributed with emotion cookies to its construction. Castles evolve and grow as more emotion cookies are added to its construction.

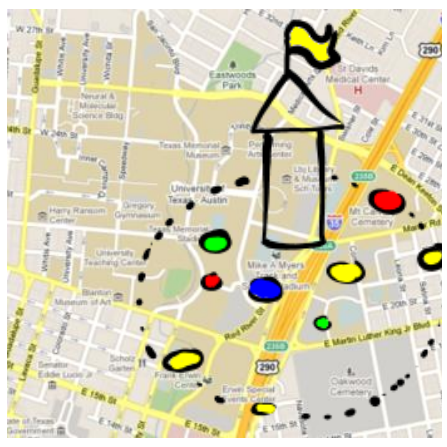

Figure 26. Emotion castle.

Each castle is ruled by a triumvirate: the Drama Queen, the Average Guy and the Zen Master. The Drama Queen is the person with the highest electrodermal activity. The Average Guy is the person that is closest to the average electrodermal activity among all players to whom the castle belongs. The Zen Master is the person with the lowest electrodermal activity. The users of the Imaginary Friend will therefore have two extra objectives in the game. One of the objectives is to become the most successful landlord and to own the greatest number of castles possible. Another objective is to belong to as many triumvirates as possible.

\section{RELATED WORK}

According to Rosalind Picard a device who knows the emotions of the person who is using it will open possibilities for new and imaginative interactions in games [12]. Some even foresee that, someday, we shall use devices that interpret our emotions and inform us of everything we need to do to be happy [13].

The Imaginary Friend is an application that is in touch with the user's emotions. Several other forms to interact with the user's emotions have already been proposed. In [14] a robot recognizes the user's affective state and displays empathic behaviors. The authors of [15] propose a computer based model that recognizes the students' affective state and reacts in such a way that learning proceeds at optimal pace. In [16] a robot mirrors the emotions happiness, fear and neutral of a human being. The How Was Your Day Companion establishes a conversation with the user about work related topics [17]. In [18] the player's facial expression of emotion controls the growth of a plant. The objective of the game is to grow the plant as fast as possible resorting to positive emotions like happiness or surprise.

One of the functionalities of the Imaginary Friend is to record and display past emotions. The Mappiness application asks users if they feel happy, relaxed or awake to discover how people's happiness is affected by their local environment [19]. The Glow mobile app shows where people are feeling good [20]. MobiMood is another mobile application that enables groups of friends to share their moods with each other [21]. In the Twitter mood project [22] the happiness of users was inferred from the analysis of their tweets. However, neither the Twitter mood project nor any of the mobile applications mentioned relate the user's emotions to the electrodermal activity.

The Imaginary Friend is projected on the floor via a pico projector connected to a mobile phone. Projectors have already been used for interacting with smart objects [23], to overcome the limitations of the small displays of mobile phones [24], to share the feelings of the user [25] or even to provide a sixth sense [26].

In what concerns the detection of emotions, the authors of [27] present a multimodal affect detector that combines conversational cues, body language, and facial features. In [28] the authors propose a method for the recognition of happiness, disgust and fear using input signals such as facial electromyograms, electrocardiogram, the respiration and the electrodermal skin response. The authors of [29] propose yet another emotion recognition system that resorts to electrocardiogram, skin temperature variation and electrodermal activity as input signals. Finally, the iCalm is a wristband that measures electrodermal activity which does not need sticky gel [30].

\section{CONCLUSIONS}

In this paper a metaphor of an Imaginary Friend and the implementation of that metaphor were presented. The Imaginary Friend walks along the user and collects the emotion cookies that user leaves behind. The user can later visualize those cookies inside a jar where the Imaginary Friend stored them (Figure 10). It is also possible to consult a list with the past emotions (Figure 11) or the places where the emotion cookies were collected (Figure 12). The Imaginary Friend resorts to variations in the electrodermal activity to know when to ask the user how she is feeling. The data obtained with the electrodermal activity sensor is transmitted via Bluetooth to the mobile phone where the Imaginary Friend's application is running. The mobile phone is connected to a pico projector that is attached to the user's backpack should strap (Figure 2 and Figure 4) and projects the Imaginary Friend on the floor. The Imaginary Friend mimics the user, walking when she is walking and standing still when the user is standing still. This behavior was implemented resorting to the accelerometer of the mobile phone.

Assuming that devices capable of monitoring electrodermal activity may, one day, become as common as mobile phones equipped with GPS and accelerometer functionality, a proposal for a multiplayer game, where users resort to their emotions to compete for the rule of emotion castles, is presented.

The interaction of the Imaginary Friend with users was tested. Results indicate that $82 \%$ felt an emotional connection with the Imaginary Friend. 63\% thought it was fun to register their emotions. Fun was, furthermore, the most frequent expression, chosen by the users, to describe the Imaginary Friend. $90 \%$ of the users also considered that the Imaginary Friend could help them reflect about their emotions and take import conclusions about their life.

The electrodermal activity was an adequate indicator to know when to question the users as $55 \%$ of them thought the Imaginary Friend questioned them the appropriate number of times. $45 \%$ thought the Imaginary Friend questioned them many times, but no user responded that the Imaginary Friend questioned her too many times.

However, 64\% of the users felt that certain emotion tags were missing among the ones presented by the Imaginary Friend. We could simply add extra emotions to the ones presented in Figure 9, but if there are too many emotion tags it will be more difficult for the user to find the one she wants. As emotion tags are differentiated by color, it will also be more difficult to find a unique color for every emotion tag. Future work will still have to be done in what concerns the emotion tags. One possibility to better organize the emotion tags is to take into consideration the arousal and valence of each emotion. 
Acknowledgements: We thank PLUX for the sensors used in this work and for all their generous help. The authors also thank everyone at IMG-CITI. This work was partly funded by FCT/MCTES, through grant SFRH/BD/61085/2009, and by Centro de Informática e Tecnologias da Informação (CITI/FCT/UNL) - 2011-2012 through grant PEstOE/EEI/UI0527/2011.

\section{REFERENCES}

[1] Hoff, E. A friend living inside me: The Forms and functions of imaginary companions. Imagination, Cognition and Personality 24, 2 (2005), 151-189.

[2] Hoff, E. Imaginary companions, creativity, and self-image in middle childhood. Creativity Research Journal 17, 2-3 (2005), 167-180.

[3] Pearson, D., Rouse, H., Doswell, S., et al. Prevalence of imaginary companions in a normal child population. Child: Care, Health and Development 27, 1 (2001), 13-22.

[4] Taylor, M., Hodges, S., and Kohányi, A. The illusion of independent agency: Do adult fiction writers experience their characters as having minds of their own? Imagination, Cognition and Personality 22, 4 (2003), 361-380.

[5] Dawson, M., Schell, A., and Diane, F. Handbook of Psychophysiology. In J. Cacioppo, L. Tassinary and G. Berntson, eds., Handbook of Psychophysiology. Cambridge University Press, Cambridge, 2000, 200-223.

[6] bioPlux research | PLUX Wireless Biosignals | Miniaturized EMG Biofeedback Systems. http://plux.info/biopluxresearch.

[7] Plutchik, R. The Nature of Emotions. American Scientist 89, 4 (2001), 344.

[8] Terwogt, M. and Hoeksma, J. Colors and emotions: preferences and combinations. Journal of General Psychology 122, 1 (1995), 5.

[9] Kaya, N. and Epps, H. Relationship between Color and Emotion: A Study of College Students. College Student Journal 38, 3 (2004), 396.

[10] Benedek, J. and Miner, T. Measuring Desirability: New methods for evaluating desirability in a usability lab setting. Proceedings of UPA Usability Professional Association 2003, 2002.

[11] Galvanic Skin Response for Measuring Emotions | Q Sensor | Affectiva. http://www.affectiva.com/q-sensor/.

[12] Picard, R. Affective Computing. M.I.T Media Laboratory Perceptual Computing Section Technical Report No. 321, 1995. http://affect.media.mit.edu/pdfs/95.picard.pdf.

[13] Drift, M. A future love story. In C. Nold, ed., Emotional Cartography - Technologies of the Self. 2009, 27.

[14] Leite, I., Pereira, A., Castellano, G., Martinho, C., Prada, R., and Paiva, A. Closing the Loop : from Affect Recognition to Empathic Interaction. Proceedings of the 3rd International Workshop on Affect Interaction in Natural Environments (AFFINE'10), ACM (2010), 43.

[15] Kort, B., Reilly, R., and Picard, R.W. An affective model of interplay between emotions and learning: reengineering educational pedagogy-building a learning companion. Proceedings IEEE International Conference on Advanced Learning Technologies 0, (2001), 43-46.
[16] Hegel, F., Spexard, T., Wrede, B., Horstmann, G., and Vogt, T. Playing a different imitation game: Interaction with an Empathic Android Robot. 2006 6th IEEERAS International Conference on Humanoid Robots, (2006), 56-61.

[17] Pulman, S.G., Boye, J., Cavazza, M., Smith, C., and Cámara, R.S. de la. 'How was your day?' Proceedings of the 2010 Workshop on Companionable Dialogue Systems, Association for Computational Linguistics (2010), 37-42.

[18] Bernhaupt, R., Boldt, A., Mirlacher, T., and Wilfinger, D. Using Emotions in Games: Emotional Flowers. Proceedings of the international conference on Advances in computer entertainment technology (ACE '07), ACM (2007), 41-48.

[19] MacKerron, G. and Mourato, S. Mappiness, the happiness mapping app. http://www.mappiness.org.uk/.

[20] Glow - How Do You Feel? http://glowapp.com/.

[21] Church, K., Hoggan, E., and Oliver, N. A study of mobile mood awareness and communication through MobiMood. Proceedings of the 6th Nordic Conference on HumanComputer Interaction: Extending Boundaries, ACM (2010), 128-137.

[22] Mislove, A., Lehmann, S., Ahn, Y.-Y., Onnela, J.-P., and Rosenquist, J.N. Pulse of the Nation: U.S. Mood Throughout the Day inferred from Twitter. 2010. http://www.ccs.neu.edu/home/amislove/twittermood/.

[23] Kawsar, F., Rukzio, E., and Kortuem, G. An explorative comparison of magic lens and personal projection for interacting with smart objects. Proceedings of the 12th international conference on Human computer interaction with mobile devices and services, ACM (2010), 157-160.

[24] Greaves, A. and Rukzio, E. View \& share: supporting copresent viewing and sharing of media using personal projection. Proceedings of the 11th International Conference on Human-Computer Interaction with Mobile Devices and Services, ACM (2009), 44:1-44:4.

[25] Ng, W.S. (Florence) and Sharlin, E. Tweeting halo: clothing that tweets. Adjunct proceedings of the 23nd annual ACM symposium on User interface software and technology, ACM (2010), 447-448.

[26] Mistry, P. and Maes, P. SixthSense: a wearable gestural interface. ACM SIGGRAPH ASIA 2009 Sketches, ACM (2009), 11:1--11:1.

[27] D’Mello, S.K. and Graesser, A. Multimodal semi-automated affect detection from conversational cues, gross body language, and facial features. User Modeling and UserAdapted Interaction 20, 2 (2010), 147-187.

[28] Rigas, G., Katsis, C.D., Ganiatsas, G., and Fotiadis, D.I. A User Independent, Biosignal Based, Emotion Recognition Method. User Modeling 2007, Springer-Verlag (2007), 314318.

[29] Kim, K.H., Bang, S.W., and Kim, S.R. Emotion recognition system using short-term monitoring of physiological signals. Medical \& Biological Engineering \& Computing 42, 3 (2004), 419-427.

[30] Hedman, E., Wilder-Smith, O., Goodwin, M.S., Poh, M.-Z., Fletcher, R., and Picard, R. iCalm: Measuring electrodermal activity in almost any setting. 2009 3rd International Conference on Affective Computing and Intelligent Interaction and Workshops, (2009), 1-2. 